\title{
Patients' perception of the roughness of restoratives
}

\author{
The in vivo perception of roughness of restorations.
}

\section{S. Jones, R. W. Billington and G. J. Pearson Br Dent J 2004; 196: 42-45}

Objective

To determine a threshold of detection value for surface roughness of restorations by patients using their tongue.

\section{Method}

Samples of composite resin were finished with differing grades of abrasive. The surface roughness $(\mathrm{Ra})$ was measured and representative scanning electro-micrographs taken. These were compared with labial enamel. Twenty-five volunteers were asked to rank them in order of perceived roughness using the tip of their tongue.

\section{Results}

These showed that the $60 \%$ of volunteers were able to rank the specimens correctly, and were able to distinguish differences in roughness values from between 0.25 and $0.50 \mu \mathrm{m}$. This range encompasses that of natural enamel.

\section{Conclusions}

The subjects were able to distinguish lower roughness values than have previously been reported. It is concluded that when finishing restorations the surface should have a maximum roughness $0.50 \mu \mathrm{m}$ if it is not to be detected by the patient.

\section{IN BRIEF}

- Patients are very aware of rough restorations.

- Little research has been carried out on the degree to which patients can detect differences in roughness.

- This study shows that previous figures for surface roughness values were much too high.

\section{COMMENT}

This study links a subjective intra oral perception of resin:ceramic composite restoration roughness to instrumental measurement, with a conclusion applicable to finishing restorations in the mouth. A composite filling material was used because of ease of handling and its recognised problems with surface finishing following any instrumentation after setting.

Discs of a hybrid composite were prepared to five grades of surface finish using silicon carbide abrasive sheets of grit size 80 , $320,800,1000$ followed by a green Shofu polishing disc, and for the smoothest, left untouched following removal of the glass slide used in specimen preparation. The surface roughness was measured using a two-dimensional profilometer and the discs given a coding from $A$ to $E$. Twenty five volunteers were then asked to fill in a questionnaire ranking them for roughness as perceived by the tip of their tongue. Photomicrographs were taken of a random sample of each roughness category and the labial surface of an extracted upper central incisor.

The greatest number of volunteers correctly placed the roughest sample and the degree of correctness fell as the samples became smoother with the lowest number at the second smoothest. This level of smoothness, a mean Ra value of $0.28 \mu \mathrm{m}$, was the finish roughness produced by the Shofu finishing disc, and that which appeared to most closely correspond with normal tooth enamel on the SEM photomicrograph. A breakdown of the incorrect rankings show that the volunteers rarely misranked by more than one place. Overall the percentage correct for individual samples was more than $75 \%$ in four of the categories. Unfortunately no information is provided concerning the size range of filler in the composite used for this study. One is therefore bound to speculate on whether the material itself could also have been a contributory factor in the results obtained.

In conclusion the authors state that patients were able to distinguish between roughness values differing by $0.5 \mu \mathrm{m}$ and that the previous norm of $20 \mu \mathrm{m}$ suggested by Van Noort in 1983 is too high.

The quality of finish required for restorations in the mouth, has always been reliant on subjective judgement by the practitioner or patient. This is a very welcome study that provides both a more scientific basis for that judgement and a useful contribution to the practicalities of day to day dentistry.

Alan Atkinson, Retired Senior Dental Officer Dental Practice Board, Hon Research Fellow Eastman Dental Institute doi:10.1038/sj.bdj.4810879 